\title{
Lung epithelial response to SARS-CoV2 and diabetes
}

\author{
Dipyaman Ganguly ${ }^{1}$ \\ ${ }^{1}$ Affiliation not available
}

May 26, 2020

\begin{abstract}
In SARS-CoV2 infections causing coronavirus disease-2019 (COVID-19) metabolic co-morbidities portend worse disease outcomes. We aimed at understanding the pathogenetic mechanisms underlying this phenomenon that may differentiate SARS$\mathrm{CoV} 2$ infection from few other respiratory viruses that caused more limited pandemics in the recent past. A meta-analysis, of publicly available gene expression studies on human lung epithelial cells (HLEC) infected with different respiratory viruses, revealed both shared and exclusive transcriptional regulations. In addition, a few key metabolic pathways were found to be exclusively enriched in SARS-CoV2 infection. Systemic metabolic disorders are known to cause similar dysregulations in HLECs, which possibly underlie the predisposition of diabetic individuals to chronic lung diseases. We further performed an integrated analysis of the transcriptome from SARS-CoV2-infected HLECs, COVID-19 bronchoalveolar lavage and autopsied lung transcriptome as well as lung transcriptome from preclinical models of diet-induced obesity, revealing significant overlaps among these key pathways in these discreet contexts. This led us to hypothesize that with underlying metabolic co-morbidities this metabolic dysregulation of the HLECs on SARS-CoV2 infection is aggravated promoting widespread disruption of lung epithelial integrity, driving the worse prognosis. We envisage that any therapeutic agent that can target these key featured pathways, viz. metformin, should be of interest in SARS-CoV2 infection and warrants further studies.
\end{abstract}

\section{Deblina Raychaudhuri ${ }^{1}$, Abhishake Lahiri ${ }^{1}$, Yogiraj Ray ${ }^{2}$, Sandip Paul ${ }^{1 *}$, Dipya- man Ganguly ${ }^{1 *}$}

\author{
${ }^{1}$ CSIR-Indian Institute of Chemical Biology, Kolkata, India. \\ ${ }^{2}$ School of Tropical Medicine, Kolkata, India. \\ *Correspondence: dipyaman@iicb.res.in, sandippaul@iicb.res.in
}

SARS-Cov2, is a newly emerged strain of coronavirus responsible for causing the coronavirus disease-2019 pandemic (COVID-19), wreaking havoc worldwide. This virus belongs to the genus Betacoronaviruswhose other members-severe acute respiratory syndrome coronavirus (SARS-CoV) and Middle East respiratory syndrome coronavirus (MERS-CoV) are known for two previous outbreaks. Although, SARS and MERS caused deadly pneumonia with mortality rates of $10 \%$ and $36 \%$ respectively $^{1}$, much higher as compared to $2.3 \%$ for SARS-CoV2 infections ${ }^{2}$, the overall number of deaths from SARS-CoV2 far outweighs the previous outbreaks. At genome level, SARS-CoV2 is more similar to a bat SARSr-CoV showing $96 \%$ genome identity than that of SARS and MERS sharing about $79 \%$ and $50 \%$ of genome identity respectively ${ }^{3,4}$. Two of the significant predictors of severity and death in patients affected by SARS, MERS and pandemic influenza are diabetes and uncontrolled glycemia ${ }^{5-7}$. Interestingly, majority of deaths in the SARS-CoV2 infection have occurred in patients suffering from metabolic co-morbidities and indeed metabolic co-morbidities have been reported to portend worse disease outcomes in multiple studies carried out in different geographical locations, 
with completely different ethnic populations. ${ }^{8-10}$ But, the reason for this often-fatal role of metabolic comorbidities, more apparent in the case of SARS-CoV2 infection, is not yet mechanistically clear. We aimed at understanding the pathogenetic mechanisms underlying this prognostic implication of metabolic disorders in COVID-19 that may differentiate SARS-CoV2 infection from few other respiratory viruses that caused more limited pandemics in the recent past.

In order to do so, we undertook a meta-analysis of three publicly available gene expression studies (RNAsequencing as well as microarray data) done on human lung epithelial cells (HLECs) (either A549 or Calu-3 cells) infected with different respiratory viruses, viz. SARS-CoV2, Respiratory Syncytial Virus or RSV, H1N1 Influenza, SARS and H3N2 Influenza ${ }^{11-13}$. The HLEC transcriptome on infection with these viruses revealed transcriptional signatures shared between the different viruses as well as transcriptional regulations exclusive to each of the viruses, as expected (Figure 1A). SARS-CoV2 was found to drive differential regulation of maximum number of genes among the datasets analyzed. Further analysis of the significantly regulated gene expressions revealed the key enriched pathways that are differentially regulated among these viruses (Figure 1B, C; Supplemental information). In Figure 1B and C, we represented pathways, among those enriched, which have role in cellular metabolic regulation and for which the nature of regulation of the pathway could be ascertained and excluded pathways that are related to diseases of no apparent relevance. Amongst these, modulation of a few key metabolic pathways (viz. AMPK signaling pathway, HIF1 $\alpha$ signaling pathway, mitophagy, AGE-RAGE signaling pathway, pentose phosphate pathway etc.) as well as the cellular senescence pathway and lysosome mediated degradation pathway were found to be exclusive to SARS-CoV2 infection (Figure 1B,C). The AGE-RAGE signaling pathway and cellular senescence pathway were upregulated (Figure 1B), whereas the AMPK signaling pathway, HIF1 $\alpha$ signaling pathway, mitophagy, pentose phosphate pathway and the lysosome pathway were all downregulated (Figure 1C). Interestingly, dysregulation of most of these metabolic pathways (upregulation of AGE-RAGE signaling and downregulation of AMPK signaling pathway, HIF1 $\alpha$ signaling pathway, mitophagy as well as the pentose phosphate pathway) have all been previously implicated in systemic disease pathogenesis observed in insulin resistance, Type 2 diabetes and its associated complications ${ }^{14-18}$.

Type 2 diabetes mellitus (T2DM) and obesity are two of the major co-morbidities portending worse prognostic outcomes in Covid-19 patients ${ }^{8-10}$. Interestingly,previous reports have established the increased association of asthma and chronic obstructive pulmonary disease(COPD) with these metabolic disorders ${ }^{19}$. Infact, these diseases associated with increased systemic metabolic disorder, are known to cause metabolic dysregulations in HLECs, which possibly underlie the predisposition of diabetic individuals towards asthma and $\mathrm{COPD}^{20,21}$. In order to compare the transcriptomic signature of SARS-Cov2 infected lung and diabetic lung, we performed an integrated analysis of the HLEC transcriptome in response to SARS-CoV2 infection as well as a COVID-19 bronchoalveolar lavage ${ }^{22}$ and autopsied lung transcriptome ${ }^{23}$ with lung transcriptome from two different datasets of preclinical rodent models of diet-induced obesity ${ }^{24,25}$ (Figure 2A). Interestingly, we found significant overlaps in how some of these key pathways are transcriptionally regulated in lung in these two distinct clinical contexts of SARS-CoV2 infection and metabolic disorder (Figure 2B). In Figure 1D, selection of pathways were based on whether 1) pathway enrichment and the nature of regulation are shared between at least two datasets, 2) are not enriched in any of the other 4 virus datasets (RSV, SARS, H1N1 and H3N2), 3) are related to cellular metabolic regulation, 4) are represented in either Figure 1B or 1C and 5) are not primarily linked to diseases of no apparent relevance. Thus, the similarity in the dysregulation of pathways in HLECs/lung upon SARS-CoV2 infection and metabolic disorders was apparent.

Our meta-analysis led us to hypothesize that with underlying metabolic co-morbidities, e.g. T2DM, this already present metabolic dysregulation of the HLECs is further aggravated upon SARS-CoV2 infection leading to faster and more widespread disruption of epithelial integrity and pulmonary damage, leading to worse disease outcomes. This provides a possible explanation for the distinction observed in the characteristics of the population subgroups worst affected by SARS-CoV2 and the other above-mentioned viruses including viruses belonging to the same family, such as SARS.

We envisage that any therapeutic agent that can target these key featured pathways, as shown in Figure 2B, 
should be of interest in SARS-CoV2 infection. This also points to a hitherto untapped potential for use of the widely used anti-diabetic drug metformin in COVID-19, which is an established modulator of most of these dysregulated pathways ${ }^{26-30}$. Indeed, metformin has been reported to inhibit AGEs, thus downregulating the AGE-RAGE signaling pathway associated with diabetes complications ${ }^{26}$. Metformin also enhances mitophagy in patients with Type 2 diabetes ${ }^{27}$. This anti-diabetic drug has also been reported to maintain cellular integrity by hindering cellular senescence ${ }^{28}$. The role of metformin in upregulation of the AMPK signaling pathway is one of its most well-established mechanisms of action involved in treating diabetes, reported in multiple studies ${ }^{29}$. Metformin has also been reported to modulate fatty acid metabolism ${ }^{30}$.

In addition to its projected effect on maintenance of lung epithelial integrity through these metabolic pathways in the epithelial cells, metformin has also been shown to boost CD8 ${ }^{+} \mathrm{T}$ cell memory though metabolic reprogramming, an effect which will also add to its potential therapeutic action in COVID-19 ${ }^{31,32}$. In a preclinical model on pulmonary effects of air pollution it was also shown that metformin can attenuate the production of the proinflammatory cytokine IL- 6 from alveolar macrophages ${ }^{33}$, which is also a critical component of the hyperimmune response in COVID- $19^{34}$. Moreover, vascular thromboembolism has been shown to be a unique feature in a number of COVID-19 patients in different studies ${ }^{35,36}$ and metformin has been shown to have an antithrombotic effect through inhibition of platelet activation and maintenance of endothelial integrity ${ }^{37,38}$. Thus, metformin, due to its multifaceted pharmacodynamics, is well poised for acting as a therapeutic agent in SARS-CoV2 infections. But for ascertaining the therapeutic potential of metformin, mechanistic studies in the context of SARS-CoV2 infection, as well as comprehensive clinical trials in COVID-19 patients with or without metabolic co-morbidities, are warranted.

\section{Methods}

\section{Datasets used in this study}

Datasets used in the meta-analysis: SARS-Cov-2 (Calu-3)- Calu-3 cells either mock-infected (control) or infected with SARS-CoV-2 (strain USA-WA1/2020), (MOI 2), were collected after 24 hours of treatment (control-3, infected-3) and subjected to RNA-seq as described in GSE147507.RSV (A549) - A549 cells either mock-infected (control) or infected with RSV (strain A2), (MOI 15), were collected after 24 hours of treatment (control-2, infected-2) and subjected to RNA-seq as described in GSE147507.H1N1 (A549) A549 cells either mock-infected (control) or infected with H1N1 (strain A/Puerto Rico/8/1934), (MOI 5), were collected after 9 hours of treatment (control-2, infected-2) and subjected to RNA-seq as described in GSE147507.SARS (Calu-3)- Calu-3 subclone 2B4 cells either mock-infected (control) or infected with SARS-CoV, (MOI 0.1), were collected after 24 hours of treatment (control-3, infected-3) and subjected to microarray as described in GSE17400.H3N2 (Calu-3) - Calu-3 cells either uninfected (control) or infected with H3N2, were collected after 24 hours of treatment (control-2, infected-3) and subjected to microarray as described in GSE19580.SARS-Cov-2 (A549)-A549 cells either mock-infected (control) or infected with SARS-CoV-2 (strain USA-WA1/2020), (MOI 0.2), were collected after 24 hours of treatment (control-3, infected-3) and subjected to RNA-seq as described in GSE147507.

The data sets used for the integrated analysis: SARS-Cov-2 (Calu-3); SARS-Cov-2 (A549), as described in previous section; Covid patient BALF- RNA-seq was performed on peripheral blood mononuclear cells (PBMCs) from 3 Covid-2019 patients and bronchoalveolar lavage fluid (BALF) from 2 of the Covid-2019 patients as described in GSA id PRJCA002326.Covid-19 lung (Autopsy)- RNA-seq was performed on a total of 15 lung sections from 5 Covid-2019 patients and 5 lung sections used as negative control as described in GSE150316. Diet induced obesity and diabetes (DIOD) rodent lung 1- RNA-seq was performed on lung tissue obtained from 3 C57BL/6J mice fed with 60\% High Fat Diet (obese mice) as well as 3 mice fed with 10\% Low Fat Diet (lean control) as described in GSE123938. Diet induced obesity and diabetes (DIOD) rodent lung 2- RNA-seq was performed on lung tissue obtained from 4 C57BL/6 mice fed with $40 \%$ High Fat Diet (obese mice) as well as 4 mice fed with 12\% Low Fat Diet (lean control) as described in GSE3963412.

\section{RNA-seq data analysis}

For the RNA-seq datasets (GSE147507, GSE123938, GSE3963412), count data was uploaded in online MeV 
software and normalised using the DESeq tool, while for GSE150316, DESeq normalised data was already available. Differential expression of genes between control and target groups was analysed using the Limma pipeline. The output provided by the Limma contained a list of statistically differentially expressed genes $(\mathrm{p}<=0.05)$. This list of genes was uploaded in the online Network Analyst software and the output containing the list of significantly enriched pathways $(\mathrm{p}<=0.05)$ was downloaded (the list of pathways for each dataset is given below), along with the list of genes implicated in each pathway. Furthermore, for each of the enriched pathways we carefully looked into the expression patterns of each member genes and based upon the directionality of the key enzymes, regulatory proteins, neighbouring genes and published studies, the upregulation/downregulation of the respective pathway was deciphered.

For the GSA id PRJCA002326 dataset, since only raw read data was available, Thefastq reads were mapped to hg38 using STAR (v2.27.2b) to create the sample-wise bam files. The bam files were then processed using Rsamtools, Rsubread and Genomic Alignments R packages to create the count table. The count data was subsequently analysed using the $\mathrm{MeV}$ software and NetworkAnalyst software as described above.

\section{Microarray data analysis}

For the microarray datasets (GSE17400, GSE19580), the data was analysed using the online Geo2R tool (default options) to determine the differential expression of genes between control and the target set and the output file containing a list of differentially expressed genes was downloaded. Amongst these genes, only those whose regulation was significant $(\mathrm{adj} . \mathrm{p}<=0.05)$ were uploaded in the NetworkAnalyst software and downstream analysis was done in an identical manner to that in the RNA-seq data analysis.

\section{Author Contributions:}

Concept and design: Ganguly, Paul, Raychaudhuri. Analysis of transcriptome data: Raychaudhuri, Lahiri, Paul. Drafting of the manuscript: Ganguly, Paul. Critical revision of the manuscript for important intellectual content: Raychaudhuri, Ray. Statistical analysis: Raychaudhuri, Lahiri, Paul.

Supervision: Ganguly, Paul.

\section{Conflict of Interest Disclosures:}

None to be reported.

\section{References}

1. Song Z, Zu Y, Bao L et al. From SARS to MERS, Thrusting Coronaviruses into the Spotlight. Viruses. 2019 Jan 14;11(1). pii: E59. doi: 10.3390/v11010059.

2. Chen Y, Li L. SARS-CoV-2: virus dynamics and host response. Lancet Infect Dis. 2020 May;20(5):515516. doi: 10.1016/S1473-3099(20)30235-8.

3. Lu R, Zhao X, Li J et al. Genomic characterisation and epidemiology of 2019 novel coronavirus: implications for virus origins and receptor binding. Lancet. 2020 Feb 22;395(10224):565-574. doi: 10.1016/S01406736(20)30251-8.

4. Zhou F, Yu T, Du R et al. Clinical course and risk factors for mortality of adult inpatients with COVID-19 in Wuhan, China: a retrospective cohort study. Lancet. 2020 Mar 28;395(10229):1054-1062. doi: 10.1016/S0140-6736(20)30566-3.

5. Yang JK, Feng Y, Yuan MY et al. Plasma glucose levels and diabetes are independent predictors for mortality and morbidity in patients with SARS. Diabet Med. 2006 Jun;23(6):623-8.doi: 10.1111/j.14645491.2006.01861.x

6. Kulcsar KA, Coleman CM, Beck SE, Frieman MB. Comorbid diabetes results in immune dysregulation and enhanced disease severity following MERS-CoV infection. JCI Insight. 2019 Oct 17;4(20). pii: 131774. doi: $10.1172 /$ jci.insight.131774. 
7. Hulme KD, Gallo LA, Short KR. Influenza Virus and Glycemic Variability in Diabetes: A Killer Combination? Front Microbiol. 2017 May 22;8:861. doi: 10.3389/fmicb.2017.00861.

8. Chen Y, Gong X, Wang L et al. Effects of hypertension, diabetes and coronary heart disease onCOVID-19 diseases severity: a systematic review and meta-analysis. medRxiv. 2020 doi: 10.1101/2020.03.25.20043133.

9. Zhu L, She ZG, Cheng X et al. Association of Blood Glucose Control and Outcomes in Patients with COVID-19 and Pre-existing Type 2 Diabetes. Cell Metab. 2020 May 1. pii: S1550-4131(20)30238-2. doi: 10.1016/j.cmet.2020.04.021.

10. Roncon L, Zuin M, Rigatelli G, Zuliani G. Diabetic patients with COVID-19 infection are at higher risk of ICU admission and poor short-term outcome. J Clin Virol. 2020 Apr 9;127:104354. doi: 10.1016/j.jcv.2020.104354.

11. Blanco-Melo D, Nilsson-Payant BE, Liu W-C et al. SARS-CoV-2 launches a unique transcriptionalsignature from in vitro, ex vivo, and in vivo systems. bioRxiv. 2020. doi: 10.1101/2020.03.24.004655.

12. Yoshikawa T, Hill TE, Yoshikawa N et al. Dynamic innate immune responses of human bronchialepithelial cells to severe acute respiratory syndrome-associated coronavirus infection. PLoS One.2010 Jan 15;5(1):e8729. doi: 10.1371/journal.pone.0008729.

13. Hsu AC, Barr I, Hansbro PM et al. Human influenza is more effective than avian influenza atantiviral suppression in airway cells. Am J Respir Cell Mol Biol. 2011 Jun;44(6):906-13. doi:10.1165/rcmb.20100157OC.

14. Ramasamy R, Yan SF, Schmidt AM. Receptor for AGE (RAGE): signaling mechanisms in the pathogenesis of diabetes and its complications. Ann N Y Acad Sci. 2011 Dec;1243:88-102. doi: 10.1111/j.17496632.2011.06320.x.

15. Coughlan KA, Valentine RJ, Ruderman NB, SahaAK. AMPK activation: a therapeutic target for type 2 diabetes? Diabetes Metab Syndr Obes. 2014 Jun 24; 7:241-53. doi: 10.2147/DMSO.S43731.

16. Xiao $\mathrm{H}, \mathrm{Gu} \mathrm{Z}$, Wang G, Zhao T. The possible mechanisms underlying the impairment of HIF- $1 \alpha$ pathway signaling in hyperglycemia and the beneficial effects of certain therapies. Int J Med Sci. 2013 Aug 22;10(10):1412-21. doi: 10.7150/ijms.5630.

17. Bhansali S, Bhansali A, Walia R, Saikia UN, Dhawan V. Alterations in Mitochondrial Oxidative Stress and Mitophagy in Subjects with Prediabetes and Type 2 Diabetes Mellitus. Front Endocrinol (Lausanne). 2017 Dec 15;8:347. doi: 10.3389/fendo.2017.00347.

18. Das UN, Rao AA.Gene expression profile in obesity and type 2 diabetes mellitus. Lipids Health Dis. 2007 Dec 14;6:35.doi: 10.1186/1476-511X-6-35.

19. Perez MK, Piedimonte G. Metabolic asthma: is there a link between obesity, diabetes, and asthma? Immunol Allergy Clin North Am. 2014 Nov;34(4):777-84. doi: 10.1016/j.iac.2014.07.002.

20. Carpaij OA, van den Berge M. The asthma-obesity relationship: underlying mechanisms andtreatment implications. Curr Opin Pulm Med. 2018 Jan;24(1):42-49. doi: 10.1097/MCP.0000000000000446.

21. Bhatraju NK, Agrawal A.Mitochondrial Dysfunction Linking Obesity and Asthma. Ann Am Thorac Soc. 2017 Nov;14(Supplement_5):S368-S373. doi: 10.1513/AnnalsATS.201701-042AW.

22. Xiong Y, Liu Y2, Cao L et al. Transcriptomic characteristics of bronchoalveolar lavage fluid andperipheral blood mononuclear cells in COVID-19 patients. Emerg Microbes Infect. 2020 Dec;9(1):761- 770. doi: 10.1080/22221751.2020.1747363.

23. Desai N, Neyaz A, Szabolcs A et al. Spectrum of Viral Load and Host Response Seen in Autopsies of SARS-CoV-2 Infected Lungs. Accession no. GSE150316, Published on GEO on May 12, 2020. 
24. Plataki M, Fan LC, Sanchez E et al. Fatty acid synthase downregulation contributes to acute lung injury in murine diet-induced obesity. JCI Insight. 2019 Jul 9;5. pii: 127823. doi: 10.1172/jci.insight.127823.

25. Siegers JY, Novakovic B, Hulme KD et al. A high fat diet increases influenza A virusassociatedcardiovascular damage. J Infect Dis. 2020 Apr 4. pii: jiaa159. doi: 10.1093/infdis/jiaa159.

26. Kumar NP, Moideen K, Nancy A et al. Systemic RAGE ligands are upregulated in tuberculosisindividuals with diabetes co-morbidity and modulated by anti-tuberculosis treatment and metformin therapy. BMC Infect Dis. 2019 Dec 9;19(1):1039. doi: 10.1186/s12879-019-4648-1.

27. Egan DF, Shackelford DB, Mihaylova MM et al. Phosphorylation of ULK1 (hATG1) by AMPactivated protein kinase connects energy sensing to mitophagy. Science. 2011 Jan 28;331(6016):456-61. doi: 10.1126/science.1196371.

28. Fang J, Yang J, Wu X et al. Metformin alleviates human cellular aging by upregulating theendoplasmic reticulum glutathione peroxidase 7. Aging Cell. 2018 Aug;17(4):e12765. doi:10.1111/acel.12765.

29. Hardie DG. AMP-activated protein kinase: maintaining energy homeostasis at the cellular andwholebody levels. Annu Rev Nutr. 2014;34:31-55. doi: 10.1146/annurev-nutr-071812-161148.

30. Tokubuchi I, Tajiri Y, Iwata Set al.Beneficial effects of metformin on energy metabolism and visceral fat volume through a possible mechanism of fatty acid oxidation in human subjects and rats. PLoS One. 2017 Feb 3;12(2):e0171293. doi: 10.1371/journal.pone.0171293.

31. Pearce EL, Walsh MC, Cejas PJ et al. Enhancing CD8 T-cell memory by modulating fatty acidmetabolism. Nature. 2009 Jul 2;460(7251):103-7. doi: 10.1038/nature08097.

32. Son J, Cho YW, Woo YJ et al. Metabolic Reprogramming by the Excessive AMPK ActivationExacerbates Antigen-Specific Memory CD8+ T Cell Differentiation after Acute Lymphocytic Choriomeningitis Virus Infection. Immune Netw. 2019 Mar 5;19(2):e11. doi: 10.4110/in.2019.19.e11.

33. Soberanes S, Misharin AV, Jairaman A et al. Metformin Targets Mitochondrial Electron Transport to Reduce Air-Pollution-Induced Thrombosis. Cell Metab. 2019 Feb 5;29(2):335-347.e5.doi: 10.1016/j.cmet.2018.09.019.

34. Merad M, Martin JC. Pathological Inflammation in Patients With COVID-19: A Key Role for Monocytes and Macrophages. Nat Rev Immunol. 2020 May 6;1-8. doi: 10.1038/s41577-020-0331-4.

35. Lodigiani C, Iapichino G, Carenzo L et al. Venous and arterial thromboembolic complications in COVID-19 patients admitted to an academic hospital in Milan, Italy. Thromb Res. 2020 Apr 23;191:9-14. doi: $10.1016 /$ j.thromres.2020.04.024.

36.Klok FA, Kruip MJHA, van der Meer NJM et al. Confirmation of the high cumulative incidence of thrombotic complications in critically ill ICU patients with COVID-19: An updated analysis. Thromb Res. 2020 Apr 30. doi: 10.1016/j.thromres.2020.04.041.

37. Xin G, Wei Z, Ji C et al. Metformin Uniquely Prevents Thrombosis by Inhibiting Platelet Activation and mtDNA Release. Sci Rep. 2016 Nov 2;6:36222. doi: 10.1038/srep36222.

38. Markowicz-Piasecka M, Huttunen KM, Broncel M, Sikora J. Sulfenamide and Sulfonamide Derivatives of Metformin - A New Option to Improve Endothelial Function and Plasma Haemostasis. Sci Rep. 2019 Apr 25;9(1):6573. doi: 10.1038/s41598-019-43083-z.

\section{Figure legend}

Figure 1. Dysregulation in lung epithelial metabolic pathways in SARS-CoV2 infection. 1A) Heatmap depicting the number of shared as well as exclusive significantly regulated genes, in the indicated cell lines in response to infection with indicated viruses. The diagonal of the heatmap represents the genes exclusively regulated only in the indicated datasets. (Detail about dataset is described in supplementary 
data).1B,C) Circos plots depicting the upregulated (B) and downregulated (C) enriched pathways that are exclusive to or shared among the indicated datasets. Datasets are the same as described in the legend of Fig 1A. 1D) Table shows the abbreviations and their corresponding pathway designations.

Figure 2. Shared enrichment of metabolic pathways between SARS-CoV2-infected and diabetic lung. 2A) Integrated analysis of the HLEC transcriptome in response to SARS-CoV2 infection as well as a COVID-19 bronchoalveolar lavage (BAL) and autopsied lung transcriptomealong with lung transcriptome from two different datasets of preclinical rodent models of diet-induced obesity and diabetes (DIOD lung) was performed. 2B) Heatmap depicting the upregulation (value 1) or downregulation (value -1) of selected pathways in the indicated datasets. The heatmap was generated using the CIMMINER software and unsupervised clustering using the correlation algorithm and average linkage cluster method.

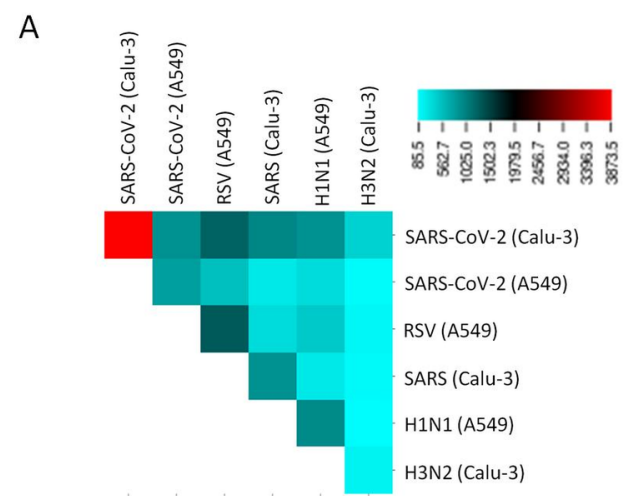

B

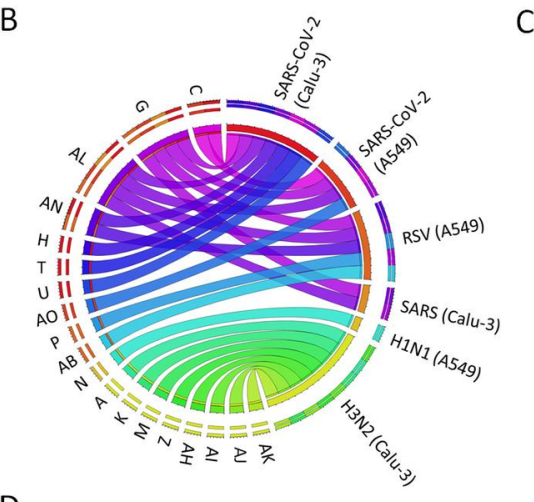

D

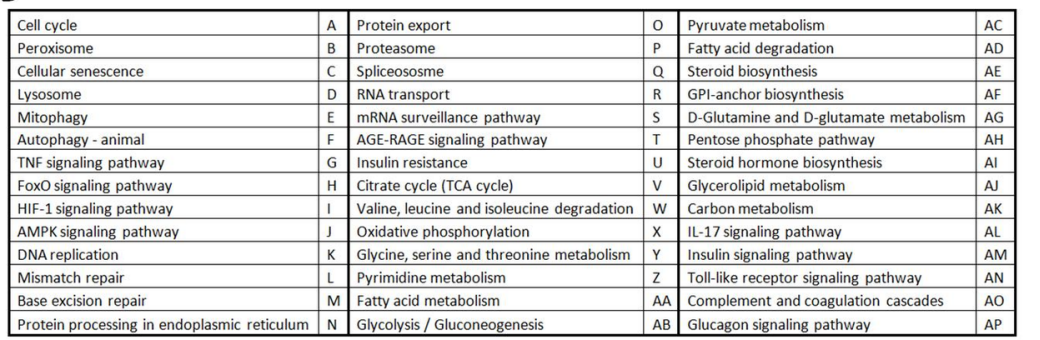

Figure 1

C

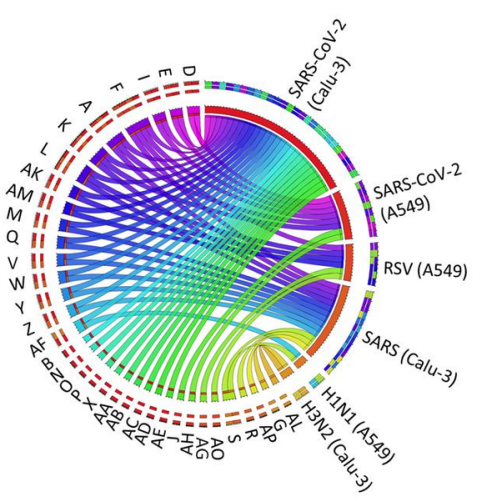
(1) 
A

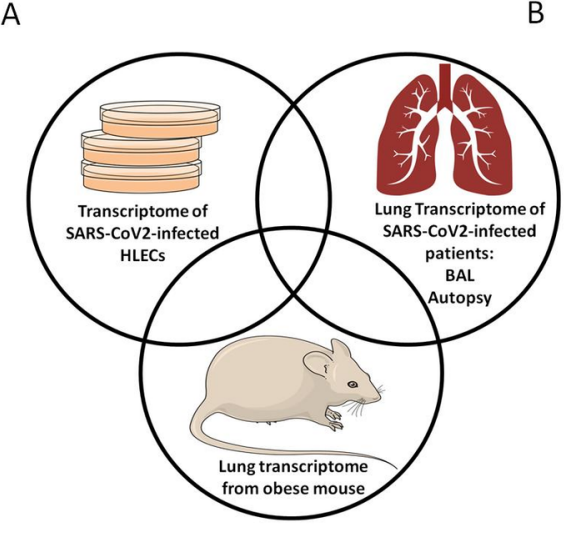

B

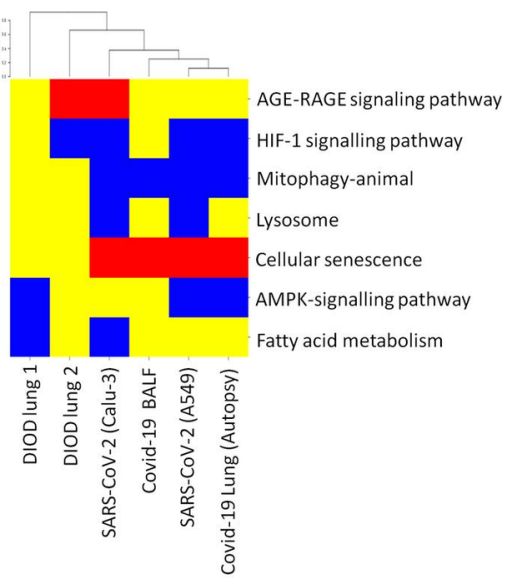

Figure 2

Supplemental Information

List of significantly enriched pathways

SARS-CoV-2 (Calu-3)- GSE147507

\begin{tabular}{llllll}
\hline Pathway & Total & Expected & Expected & Hits & Hits \\
\hline TNF signaling pathway & 110 & 42.9 & 80 & 80 & $5.09 \mathrm{E}$ \\
Non-alcoholic fatty liver disease (NAFLD) & 149 & 58.1 & 99 & 99 & $7.23 \mathrm{E}$ \\
Metabolic pathways & 1430 & 558 & 670 & 670 & $1.40 \mathrm{E}$ \\
Herpes simplex infection & 492 & 192 & 261 & 261 & $4.63 \mathrm{E}$ \\
Pancreatic cancer & 75 & 29.2 & 57 & 57 & $6.33 \mathrm{E}$ \\
HTLV-I infection & 219 & 85.4 & 130 & 130 & $5.07 \mathrm{E}$ \\
Hepatitis C & 155 & 60.4 & 98 & 98 & $6.30 \mathrm{E}$ \\
Epstein-Barr virus infection & 201 & 78.4 & 120 & 120 & $1.46 \mathrm{E}$ \\
Cell cycle & 124 & 48.3 & 81 & 81 & $2.06 \mathrm{E}$ \\
Colorectal cancer & 86 & 33.5 & 60 & 60 & $6.08 \mathrm{E}$ \\
DNA replication & 36 & 14 & 31 & 31 & $6.79 \mathrm{E}$ \\
Hepatitis B & 163 & 63.5 & 98 & 98 & $2.87 \mathrm{E}$ \\
Apoptosis & 136 & 53 & 84 & 84 & $5.10 \mathrm{E}$ \\
Chronic myeloid leukemia & 76 & 29.6 & 52 & 52 & $1.73 \mathrm{E}$ \\
Influenza A & 167 & 65.1 & 96 & 96 & $7.79 \mathrm{E}$ \\
Huntington's disease & 193 & 75.2 & 108 & 108 & $1.00 \mathrm{E}$ \\
Small cell lung cancer & 93 & 36.3 & 59 & 59 & $1.40 \mathrm{E}$ \\
Prostate cancer & 97 & 37.8 & 61 & 61 & $1.44 \mathrm{E}$ \\
Parkinson's disease & 142 & 55.4 & 83 & 83 & $1.72 \mathrm{E}$ \\
AGE-RAGE signaling pathway in diabetic complications & 100 & 39 & 62 & 62 & $2.38 \mathrm{E}$ \\
Endometrial cancer & 58 & 22.6 & 40 & 40 & $3.38 \mathrm{E}$ \\
Non-small cell lung cancer & 66 & 25.7 & 44 & 44 & $4.53 \mathrm{E}$ \\
Kaposi's sarcoma-associated herpesvirus infection & 186 & 72.5 & 102 & 102 & $6.78 \mathrm{E}$ \\
Measles & 138 & 53.8 & 79 & 79 & $9.06 \mathrm{E}$ \\
& & & &
\end{tabular}




\begin{tabular}{|c|c|c|c|c|c|}
\hline Pathway & Total & Expected & Expected & Hits & Hits \\
\hline Platinum drug resistance & 73 & 28.5 & 47 & 47 & 9.1 \\
\hline Alzheimer's disease & 171 & 66.7 & 94 & 94 & 1.3 \\
\hline Endocrine resistance & 98 & 38.2 & 59 & 59 & 1.5 \\
\hline Pathways in cancer & 530 & 207 & 252 & 252 & 2.0 \\
\hline Glioma & 75 & 29.2 & 47 & 47 & 2.6 \\
\hline Valine, leucine and isoleucine degradation & 48 & 18.7 & 33 & 33 & 2.7 \\
\hline Tuberculosis & 179 & 69.8 & 96 & 96 & 4.1 \\
\hline Ubiquitin mediated proteolysis & 137 & 53.4 & 76 & 76 & 5.8 \\
\hline Oxidative phosphorylation & 133 & 51.8 & 74 & 74 & 6.4 \\
\hline Base excision repair & 33 & 12.9 & 24 & 24 & 8.5 \\
\hline Endocytosis & 244 & 95.1 & 124 & 124 & 9.1 \\
\hline Autophagy - animal & 128 & 49.9 & 71 & 71 & 0.0 \\
\hline p53 signaling pathway & 72 & 28.1 & 44 & 44 & 0.0 \\
\hline Glyoxylate and dicarboxylate metabolism & 30 & 11.7 & 22 & 22 & 0.00 \\
\hline Mismatch repair & 23 & 8.97 & 18 & 18 & 0.0 \\
\hline EGFR tyrosine kinase inhibitor resistance & 79 & 30.8 & 47 & 47 & 0.0 \\
\hline Neurotrophinsignaling pathway & 119 & 46.4 & 66 & 66 & 0.0 \\
\hline Legionellosis & 55 & 21.4 & 35 & 35 & 0.0 \\
\hline B cell receptor signaling pathway & 71 & 27.7 & 43 & 43 & 0.0 \\
\hline FoxOsignaling pathway & 132 & 51.5 & 72 & 72 & 0.0 \\
\hline NOD-like receptor signaling pathway & 178 & 69.4 & 93 & 93 & 0.0 \\
\hline Fluid shear stress and atherosclerosis & 139 & 54.2 & 75 & 75 & 0.0 \\
\hline Chagas disease (American trypanosomiasis) & 103 & 40.2 & 58 & 58 & 0.0 \\
\hline Progesterone-mediated oocyte maturation & 99 & 38.6 & 56 & 56 & 0.0 \\
\hline IL-17 signaling pathway & 93 & 36.3 & 53 & 53 & 0.000 \\
\hline ErbBsignaling pathway & 85 & 33.1 & 49 & 49 & 0.000 \\
\hline Protein processing in endoplasmic reticulum & 165 & 64.3 & 86 & 86 & 0.00 \\
\hline HIF-1 signaling pathway & 100 & 39 & 56 & 56 & 0.000 \\
\hline Mitophagy - animal & 65 & 25.3 & 39 & 39 & 0.000 \\
\hline Central carbon metabolism in cancer & 65 & 25.3 & 39 & 39 & 0.000 \\
\hline Carbon metabolism & 116 & 45.2 & 63 & 63 & 0.000 \\
\hline Pertussis & 76 & 29.6 & 44 & 44 & 0.000 \\
\hline Prolactin signaling pathway & 70 & 27.3 & 41 & 41 & 0.000 \\
\hline Osteoclast differentiation & 128 & 49.9 & 68 & 68 & 0.000 \\
\hline Toxoplasmosis & 113 & 44 & 61 & 61 & 0.000 \\
\hline mTORsignaling pathway & 153 & 59.6 & 79 & 79 & 0.000 \\
\hline Nucleotide excision repair & 47 & 18.3 & 29 & 29 & 0.001 \\
\hline Proteasome & 45 & 17.5 & 28 & 28 & 0.001 \\
\hline Melanoma & 72 & 28.1 & 41 & 41 & 0.001 \\
\hline NF-kappa B signaling pathway & 100 & 39 & 54 & 54 & 0.001 \\
\hline Epithelial cell signaling in Helicobacter pylori infection & 68 & 26.5 & 39 & 39 & 0.001 \\
\hline Acute myeloid leukemia & 66 & 25.7 & 38 & 38 & 0.001 \\
\hline Viral carcinogenesis & 201 & 78.4 & 99 & 99 & 0.00 \\
\hline Breast cancer & 147 & 57.3 & 75 & 75 & 0.00 \\
\hline Citrate cycle (TCA cycle) & 30 & 11.7 & 20 & 20 & 0.00 \\
\hline Vibrio cholerae infection & 50 & 19.5 & 30 & 30 & 0.00 \\
\hline Lysosome & 123 & 47.9 & 64 & 64 & 0.00 \\
\hline Notch signaling pathway & 48 & 18.7 & 29 & 29 & 0.00 \\
\hline Hippo signaling pathway & 154 & 60 & 77 & 77 & 0.00 \\
\hline Tight junction & 170 & 66.3 & 84 & 84 & 0.00 \\
\hline
\end{tabular}




\begin{tabular}{|c|c|c|c|c|c|}
\hline Pathway & Total & Expected & Expected & Hits & Hits \\
\hline Bladder cancer & 41 & 16 & 25 & 25 & 0.003 \\
\hline Apoptosis - multiple species & 33 & 12.9 & 21 & 21 & 0.003 \\
\hline Thyroid cancer & 37 & 14.4 & 23 & 23 & 0.003 \\
\hline Longevity regulating pathway - multiple species & 62 & 24.2 & 35 & 35 & 0.003 \\
\hline Biosynthesis of amino acids & 75 & 29.2 & 41 & 41 & .004 \\
\hline Renal cell carcinoma & 69 & 26.9 & 38 & 38 & $.004^{\prime}$ \\
\hline Toll-like receptor signaling pathway & 104 & 40.5 & 54 & 54 & $0.004^{\prime}$ \\
\hline Glycosaminoglycan biosynthesis - chondroitin sulfate / dermatan sulfate & 20 & 7.8 & 14 & 14 & 0.004 \\
\hline Estrogensignaling pathway & 138 & 53.8 & 69 & 69 & .005 \\
\hline Terpenoid backbone biosynthesis & 22 & 8.58 & 15 & 15 & .005 \\
\hline Glycine, serine and threonine metabolism & 40 & 15.6 & 24 & 24 & .005 \\
\hline Spliceosome & 134 & 52.2 & 67 & 67 & .005 \\
\hline Oocyte meiosis & 125 & 48.7 & 63 & 63 & .005 \\
\hline VEGF signaling pathway & 59 & 23 & 33 & 33 & 0.005 \\
\hline Adherens junction & 72 & 28.1 & 39 & 39 & 0.006 \\
\hline Pyrimidine metabolism & 57 & 22.2 & 32 & 32 & 0.006 \\
\hline Proteoglycans in cancer & 201 & 78.4 & 96 & 96 & 0.006 \\
\hline Pathogenic Escherichia coli infection & 55 & 21.4 & 31 & 31 & 0.006 \\
\hline MAPK signaling pathway & 295 & 115 & 136 & 136 & 0.006 \\
\hline Fatty acid metabolism & 53 & 20.7 & 30 & 30 & 0.006 \\
\hline Fc gamma R-mediated phagocytosis & 91 & 35.5 & 47 & 47 & 0.009 \\
\hline N-Glycan biosynthesis & 50 & 19.5 & 28 & 28 & 0.010 \\
\hline Cellular senescence & 160 & 62.4 & 77 & 77 & 0.010 \\
\hline Fatty acid degradation & 44 & 17.2 & 25 & 25 & 0.012 \\
\hline Glycolysis / Gluconeogenesis & 68 & 26.5 & 36 & 36 & 0.013 \\
\hline Fc epsilon RI signaling pathway & 68 & 26.5 & 36 & 36 & 0.013 \\
\hline Alanine, aspartate and glutamate metabolism & 36 & 14 & 21 & 21 & 0.014 \\
\hline Signaling pathways regulating pluripotency of stem cells & 139 & 54.2 & 67 & 67 & 0.016 \\
\hline 2-Oxocarboxylic acid metabolism & 18 & 7.02 & 12 & 12 & 0.016 \\
\hline One carbon pool by folate & 20 & 7.8 & 13 & 13 & 0.016 \\
\hline Pyruvate metabolism & 39 & 15.2 & 22 & 22 & 0.020 \\
\hline Amino sugar and nucleotide sugar metabolism & 48 & 18.7 & 26 & 26 & 0.023 \\
\hline Antifolate resistance & 31 & 12.1 & 18 & 18 & 0.024 \\
\hline GnRHsignaling pathway & 93 & 36.3 & 46 & 46 & 0.024 \\
\hline Wntsignaling pathway & 158 & 61.6 & 74 & 74 & 0.025 \\
\hline Insulin signaling pathway & 137 & 53.4 & 65 & 65 & 0.025 \\
\hline Regulation of lipolysis in adipocytes & 55 & 21.4 & 29 & 29 & 0.02 \\
\hline Glycosylphosphatidylinositol (GPI)-anchor biosynthesis & 25 & 9.75 & 15 & 15 & 0.02 \\
\hline Tryptophan metabolism & 42 & 16.4 & 23 & 23 & 0.02 \\
\hline Protein export & 23 & 8.97 & 14 & 14 & 0.02 \\
\hline Longevity regulating pathway & 89 & 34.7 & 44 & 44 & 0.02 \\
\hline Steroid biosynthesis & 19 & 7.41 & 12 & 12 & 0.02 \\
\hline Sphingolipid signaling pathway & 119 & 46.4 & 57 & 57 & 0.02 \\
\hline Insulin resistance & 108 & 42.1 & 52 & 52 & 0.0 \\
\hline Peroxisome & 83 & 32.4 & 41 & 41 & 0.0 \\
\hline Inositol phosphate metabolism & 74 & 28.8 & 37 & 37 & 0.0 \\
\hline
\end{tabular}

SARS-CoV-2 (A549)- GSE147507 


\begin{tabular}{llllll}
\hline Pathway & Total & Expected & Hits & P.Value & FDR \\
\hline NOD-like receptor signaling pathway & 178 & 16.9 & 33 & 0.000121 & 0.0318 \\
Pertussis & 76 & 7.21 & 18 & $2.00 \mathrm{E}-04$ & 0.0318 \\
Autophagy - animal & 128 & 12.1 & 24 & 0.000836 & 0.0886 \\
Leishmaniasis & 74 & 7.02 & 16 & 0.00129 & 0.102 \\
IL-17 signaling pathway & 93 & 8.82 & 18 & 0.0025 & 0.134 \\
Lysosome & 123 & 11.7 & 22 & 0.00253 & 0.134 \\
Amoebiasis & 96 & 9.11 & 18 & 0.00358 & 0.163 \\
Calcium signaling pathway & 188 & 17.8 & 29 & 0.00573 & 0.228 \\
TNF signaling pathway & 110 & 10.4 & 19 & 0.00711 & 0.243 \\
Influenza A & 167 & 15.8 & 26 & 0.00764 & 0.243 \\
Complement and coagulation cascades & 79 & 7.49 & 14 & 0.0156 & 0.427 \\
Phagosome & 152 & 14.4 & 23 & 0.0161 & 0.427 \\
Pentose phosphate pathway & 30 & 2.85 & 7 & 0.0197 & 0.481 \\
PI3K-Akt signaling pathway & 354 & 33.6 & 45 & 0.0247 & 0.505 \\
Staphylococcus aureus infection & 68 & 6.45 & 12 & 0.0248 & 0.505 \\
HIF-1 signaling pathway & 100 & 9.49 & 16 & 0.0254 & 0.505 \\
Apoptosis & 136 & 12.9 & 20 & 0.0313 & 0.538 \\
Legionellosis & 55 & 5.22 & 10 & 0.0321 & 0.538 \\
AMPK signaling pathway & 120 & 11.4 & 18 & 0.0334 & 0.538 \\
EGFR tyrosine kinase inhibitor resistance & 79 & 7.49 & 13 & 0.0338 & 0.538 \\
Measles & 138 & 13.1 & 20 & 0.0358 & 0.539 \\
Epstein-Barr virus infection & 201 & 19.1 & 27 & 0.0398 & 0.539 \\
Mitophagy - animal & 65 & 6.17 & 11 & 0.0406 & 0.539 \\
Endocrine resistance & 98 & 9.3 & 15 & 0.0422 & 0.539 \\
Cell cycle & 124 & 11.8 & 18 & 0.0442 & 0.539 \\
Cellular senescence & 160 & 15.2 & 22 & 0.0478 & 0.539 \\
DNA replication & 36 & 3.42 & 7 & 0.0493 & 0.539 \\
\hline
\end{tabular}

RSV (A549)- GSE147507

\begin{tabular}{lllllll}
\hline Pathway & Total & Expected & Expected & Hits & P.Value & FDR \\
\hline Influenza A & 167 & 23 & 46 & 46 & $1.78 \mathrm{E}-06$ & 0.000565 \\
Epstein-Barr virus infection & 201 & 27.7 & 50 & 50 & $1.51 \mathrm{E}-05$ & 0.00229 \\
Legionellosis & 55 & 7.58 & 20 & 20 & $2.16 \mathrm{E}-05$ & 0.00229 \\
Kaposi's sarcoma-associated herpesvirus infection & 186 & 25.7 & 46 & 46 & $3.85 \mathrm{E}-05$ & 0.00279 \\
Hepatitis C & 155 & 21.4 & 40 & 40 & $4.38 \mathrm{E}-05$ & 0.00279 \\
Small cell lung cancer & 93 & 12.8 & 27 & 27 & $9.07 \mathrm{E}-05$ & 0.00481 \\
Bladder cancer & 41 & 5.65 & 15 & 15 & 0.000212 & 0.00877 \\
Platinum drug resistance & 73 & 10.1 & 22 & 22 & 0.000221 & 0.00877 \\
Complement and coagulation cascades & 79 & 10.9 & 23 & 23 & 0.000282 & 0.00996 \\
TNF signaling pathway & 110 & 15.2 & 29 & 29 & 0.000324 & 0.0103 \\
Measles & 138 & 19 & 34 & 34 & 0.000413 & 0.011 \\
Chronic myeloid leukemia & 76 & 10.5 & 22 & 22 & 0.000415 & 0.011 \\
Chemokine signaling pathway & 190 & 26.2 & 43 & 43 & 0.000556 & 0.0136 \\
Pathways in cancer & 530 & 73.1 & 99 & 99 & 0.000689 & 0.0156 \\
Glioma & 75 & 10.3 & 21 & 21 & 0.000902 & 0.0191 \\
Apoptosis - multiple species & 33 & 4.55 & 12 & 12 & 0.000964 & 0.0192 \\
Non-small cell lung cancer & 66 & 9.1 & 19 & 19 & 0.00108 & 0.0203 \\
Apoptosis & 136 & 18.8 & 32 & 32 & 0.00139 & 0.0245
\end{tabular}




\begin{tabular}{|c|c|c|c|c|c|c|}
\hline Pathway & Total & Expected & Expected & Hits & P.Value & FDR \\
\hline Rheumatoid arthritis & 91 & 12.5 & 23 & 23 & 0.00237 & 0.0397 \\
\hline Type II diabetes mellitus & 46 & 6.34 & 14 & 14 & 0.0027 & 0.041 \\
\hline Focal adhesion & 199 & 27.4 & 42 & 42 & 0.00271 & 0.041 \\
\hline Melanoma & 72 & 9.93 & 19 & 19 & 0.00328 & 0.0474 \\
\hline EGFR tyrosine kinase inhibitor resistance & 79 & 10.9 & 20 & 20 & 0.00435 & 0.0601 \\
\hline ErbBsignaling pathway & 85 & 11.7 & 21 & 21 & 0.0048 & 0.0636 \\
\hline Glyoxylate and dicarboxylate metabolism & 30 & 4.14 & 10 & 10 & 0.00523 & 0.0659 \\
\hline Colorectal cancer & 86 & 11.9 & 21 & 21 & 0.00555 & 0.0659 \\
\hline Prion diseases & 35 & 4.83 & 11 & 11 & 0.00574 & 0.0659 \\
\hline Central carbon metabolism in cancer & 65 & 8.96 & 17 & 17 & 0.0058 & 0.0659 \\
\hline p53 signaling pathway & 72 & 9.93 & 18 & 18 & 0.00762 & 0.0835 \\
\hline Hepatitis B & 163 & 22.5 & 34 & 34 & 0.00789 & 0.0837 \\
\hline Mismatch repair & 23 & 3.17 & 8 & 8 & 0.00918 & 0.0941 \\
\hline Insulin signaling pathway & 137 & 18.9 & 29 & 29 & 0.011 & 0.109 \\
\hline Viral myocarditis & 59 & 8.14 & 15 & 15 & 0.0121 & 0.117 \\
\hline Prolactin signaling pathway & 70 & 9.65 & 17 & 17 & 0.0126 & 0.117 \\
\hline IL-17 signaling pathway & 93 & 12.8 & 21 & 21 & 0.0139 & 0.124 \\
\hline Carbon metabolism & 116 & 16 & 25 & 25 & 0.014 & 0.124 \\
\hline Osteoclast differentiation & 128 & 17.7 & 27 & 27 & 0.0145 & 0.124 \\
\hline Endocytosis & 244 & 33.6 & 46 & 46 & 0.0153 & 0.128 \\
\hline Proteasome & 45 & 6.21 & 12 & 12 & 0.0162 & 0.132 \\
\hline Glutathione metabolism & 56 & 7.72 & 14 & 14 & 0.0174 & 0.136 \\
\hline NOD-like receptor signaling pathway & 178 & 24.5 & 35 & 35 & 0.0175 & 0.136 \\
\hline Glycolysis / Gluconeogenesis & 68 & 9.38 & 16 & 16 & 0.0204 & 0.154 \\
\hline D-Glutamine and D-glutamate metabolism & 5 & 0.69 & 3 & 3 & 0.0211 & 0.156 \\
\hline Renal cell carcinoma & 69 & 9.52 & 16 & 16 & 0.0232 & 0.168 \\
\hline Endocrine resistance & 98 & 13.5 & 21 & 21 & 0.0244 & 0.172 \\
\hline PI3K-Akt signaling pathway & 354 & 48.8 & 62 & 62 & 0.0253 & 0.175 \\
\hline Pertussis & 76 & 10.5 & 17 & 17 & 0.0275 & 0.186 \\
\hline B cell receptor signaling pathway & 71 & 9.79 & 16 & 16 & 0.0299 & 0.198 \\
\hline Platelet activation & 124 & 17.1 & 25 & 25 & 0.0305 & 0.198 \\
\hline Vasopressin-regulated water reabsorption & 44 & 6.07 & 11 & 11 & 0.033 & 0.21 \\
\hline Amoebiasis & 96 & 13.2 & 20 & 20 & 0.0363 & 0.226 \\
\hline Viral carcinogenesis & 201 & 27.7 & 37 & 37 & 0.0381 & 0.226 \\
\hline Regulation of actin cytoskeleton & 214 & 29.5 & 39 & 39 & 0.039 & 0.226 \\
\hline Fc gamma R-mediated phagocytosis & 91 & 12.5 & 19 & 19 & 0.0398 & 0.226 \\
\hline Hematopoietic cell lineage & 97 & 13.4 & 20 & 20 & 0.04 & 0.226 \\
\hline Ferroptosis & 40 & 5.52 & 10 & 10 & 0.0411 & 0.226 \\
\hline Fc epsilon RI signaling pathway & 68 & 9.38 & 15 & 15 & 0.0413 & 0.226 \\
\hline Staphylococcus aureus infection & 68 & 9.38 & 15 & 15 & 0.0413 & 0.226 \\
\hline Autophagy - animal & 128 & 17.7 & 25 & 25 & 0.043 & 0.232 \\
\hline Toll-like receptor signaling pathway & 104 & 14.3 & 21 & 21 & 0.044 & 0.233 \\
\hline Pancreatic cancer & 75 & 10.3 & 16 & 16 & 0.0472 & 0.242 \\
\hline Tuberculosis & 179 & 24.7 & 33 & 33 & 0.0472 & 0.242 \\
\hline
\end{tabular}

SARS (Calu-3)- GSE17400

\begin{tabular}{lllllll}
\hline Pathway & Total & Expected & Expected & Hits & P.Value & FDR \\
\hline Cell cycle & 124 & 16.3 & 40 & 40 & $2.18 \mathrm{E}-08$ & $6.92 \mathrm{E}-06$
\end{tabular}




\begin{tabular}{|c|c|c|c|c|c|c|}
\hline$\overline{\text { Pathway }}$ & Total & Expected & Expected & Hits & P.Value & FDR \\
\hline Oocyte meiosis & 125 & 16.4 & 36 & 36 & $2.41 \mathrm{E}-06$ & 0.000383 \\
\hline DNA replication & 36 & 4.72 & 15 & 15 & $1.97 \mathrm{E}-05$ & 0.00209 \\
\hline One carbon pool by folate & 20 & 2.62 & 10 & 10 & $7.61 \mathrm{E}-05$ & 0.00605 \\
\hline Mismatch repair & 23 & 3.01 & 9 & 9 & 0.00161 & 0.102 \\
\hline Amphetamine addiction & 68 & 8.91 & 18 & 18 & 0.00228 & 0.12 \\
\hline Neurotrophinsignaling pathway & 119 & 15.6 & 27 & 27 & 0.00265 & 0.12 \\
\hline Biosynthesis of amino acids & 75 & 9.83 & 19 & 19 & 0.00301 & 0.12 \\
\hline Progesterone-mediated oocyte maturation & 99 & 13 & 23 & 23 & 0.00388 & 0.125 \\
\hline Spliceosome & 134 & 17.6 & 29 & 29 & 0.00393 & 0.125 \\
\hline Long-term potentiation & 67 & 8.78 & 17 & 17 & 0.00479 & 0.133 \\
\hline Melanogenesis & 101 & 13.2 & 23 & 23 & 0.00505 & 0.133 \\
\hline Pyrimidine metabolism & 57 & 7.47 & 15 & 15 & 0.00545 & 0.133 \\
\hline RNA transport & 165 & 21.6 & 33 & 33 & 0.00786 & 0.179 \\
\hline Legionellosis & 55 & 7.21 & 14 & 14 & 0.00971 & 0.205 \\
\hline Dopaminergic synapse & 131 & 17.2 & 27 & 27 & 0.0103 & 0.205 \\
\hline Amoebiasis & 96 & 12.6 & 21 & 21 & 0.0115 & 0.206 \\
\hline Citrate cycle (TCA cycle) & 30 & 3.93 & 9 & 9 & 0.0121 & 0.206 \\
\hline mRNA surveillance pathway & 91 & 11.9 & 20 & 20 & 0.0127 & 0.206 \\
\hline Glucagon signaling pathway & 103 & 13.5 & 22 & 22 & 0.013 & 0.206 \\
\hline TNF signaling pathway & 110 & 14.4 & 23 & 23 & 0.0144 & 0.206 \\
\hline Alanine, aspartate and glutamate metabolism & 36 & 4.72 & 10 & 10 & 0.0147 & 0.206 \\
\hline Renin secretion & 69 & 9.04 & 16 & 16 & 0.0149 & 0.206 \\
\hline Nucleotide excision repair & 47 & 6.16 & 12 & 12 & 0.0157 & 0.208 \\
\hline HTLV-I infection & 219 & 28.7 & 40 & 40 & 0.0171 & 0.218 \\
\hline Gap junction & 88 & 11.5 & 19 & 19 & 0.0179 & 0.219 \\
\hline Signaling pathways regulating pluripotency of stem cells & 139 & 18.2 & 27 & 27 & 0.0218 & 0.251 \\
\hline Base excision repair & 33 & 4.32 & 9 & 9 & 0.0227 & 0.251 \\
\hline 2-Oxocarboxylic acid metabolism & 18 & 2.36 & 6 & 6 & 0.0229 & 0.251 \\
\hline Circadian entrainment & 97 & 12.7 & 20 & 20 & 0.0248 & 0.263 \\
\hline IL-17 signaling pathway & 93 & 12.2 & 19 & 19 & 0.0308 & 0.303 \\
\hline GnRHsignaling pathway & 93 & 12.2 & 19 & 19 & 0.0308 & 0.303 \\
\hline Ubiquitin mediated proteolysis & 137 & 18 & 26 & 26 & 0.0315 & 0.303 \\
\hline Kaposi's sarcoma-associated herpesvirus infection & 186 & 24.4 & 33 & 33 & 0.041 & 0.351 \\
\hline Autophagy - animal & 128 & 16.8 & 24 & 24 & 0.0427 & 0.351 \\
\hline Osteoclast differentiation & 128 & 16.8 & 24 & 24 & 0.0427 & 0.351 \\
\hline Valine, leucine and isoleucine degradation & 48 & 6.29 & 11 & 11 & 0.0428 & 0.351 \\
\hline Fanconianemia pathway & 54 & 7.08 & 12 & 12 & 0.0437 & 0.351 \\
\hline p53 signaling pathway & 72 & 9.44 & 15 & 15 & 0.044 & 0.351 \\
\hline Chagas disease (American trypanosomiasis) & 103 & 13.5 & 20 & 20 & 0.0442 & 0.351 \\
\hline EGFR tyrosine kinase inhibitor resistance & 79 & 10.4 & 16 & 16 & 0.0482 & 0.37 \\
\hline Cocaine addiction & 49 & 6.42 & 11 & 11 & 0.0489 & 0.37 \\
\hline
\end{tabular}

\section{H1N1 (A549)- GSE147507}

\begin{tabular}{llllll}
\hline Pathway & Total & Expected & Hits & P.Value & FDR \\
\hline Protein processing in endoplasmic reticulum & 165 & 17.2 & 33 & 0.000163 & 0.0518 \\
Legionellosis & 55 & 5.74 & 14 & 0.00118 & 0.188 \\
TGF-beta signaling pathway & 92 & 9.6 & 17 & 0.0133 & 1 \\
Cytokine-cytokine receptor interaction & 294 & 30.7 & 42 & 0.0208 & 1
\end{tabular}




\begin{tabular}{llllll}
\hline Pathway & Total & Expected & Hits & P.Value & FDR \\
\hline Protein digestion and absorption & 90 & 9.39 & 16 & 0.0227 & 1 \\
Amino sugar and nucleotide sugar metabolism & 48 & 5.01 & 10 & 0.024 & 1 \\
mRNA surveillance pathway & 91 & 9.49 & 16 & 0.0249 & 1 \\
Selenocompound metabolism & 17 & 1.77 & 5 & 0.026 & 1 \\
Glycosylphosphatidylinositol (GPI)-anchor biosynthesis & 25 & 2.61 & 6 & 0.0397 & 1 \\
Endocytosis & 244 & 25.5 & 34 & 0.0475 & 1 \\
\hline
\end{tabular}

H3N2 (Calu-3)-GSE19580

\begin{tabular}{|c|c|c|c|c|c|c|}
\hline Pathway & Total & Expected & Expected & Hits & P.Value & FDR \\
\hline DNA replication & 36 & 1.51 & 12 & 12 & $1.24 \mathrm{E}-08$ & $3.96 \mathrm{E}-06$ \\
\hline Cell cycle & 124 & 5.21 & 20 & 20 & $1.83 \mathrm{E}-07$ & $2.91 \mathrm{E}-05$ \\
\hline Pentose phosphate pathway & 30 & 1.26 & 9 & 9 & $2.39 \mathrm{E}-06$ & 0.000253 \\
\hline Epstein-Barr virus infection & 201 & 8.44 & 22 & 22 & $3.36 \mathrm{E}-05$ & 0.00267 \\
\hline Pentose and glucuronate interconversions & 34 & 1.43 & 8 & 8 & $6.20 \mathrm{E}-05$ & 0.00394 \\
\hline Hepatitis C & 155 & 6.51 & 17 & 17 & 0.000257 & 0.0121 \\
\hline Biosynthesis of amino acids & 75 & 3.15 & 11 & 11 & 0.000267 & 0.0121 \\
\hline Fructose and mannose metabolism & 33 & 1.39 & 7 & 7 & 0.000359 & 0.0143 \\
\hline Hepatitis B & 163 & 6.85 & 17 & 17 & 0.000467 & 0.0153 \\
\hline IL-17 signaling pathway & 93 & 3.91 & 12 & 12 & 0.00048 & 0.0153 \\
\hline Carbon metabolism & 116 & 4.87 & 13 & 13 & 0.0011 & 0.0319 \\
\hline Salmonella infection & 86 & 3.61 & 10 & 10 & 0.00309 & 0.0798 \\
\hline Necroptosis & 162 & 6.8 & 15 & 15 & 0.00326 & 0.0798 \\
\hline Pathways in cancer & 530 & 22.3 & 35 & 35 & 0.0048 & 0.106 \\
\hline Kaposi's sarcoma-associated herpesvirus infection & 186 & 7.81 & 16 & 16 & 0.005 & 0.106 \\
\hline TNF signaling pathway & 110 & 4.62 & 11 & 11 & 0.0063 & 0.125 \\
\hline NOD-like receptor signaling pathway & 178 & 7.48 & 15 & 15 & 0.00779 & 0.146 \\
\hline Glutathione metabolism & 56 & 2.35 & 7 & 7 & 0.00855 & 0.147 \\
\hline Galactose metabolism & 31 & 1.3 & 5 & 5 & 0.00876 & 0.147 \\
\hline Viral carcinogenesis & 201 & 8.44 & 16 & 16 & 0.0103 & 0.16 \\
\hline HTLV-I infection & 219 & 9.2 & 17 & 17 & 0.0106 & 0.16 \\
\hline Tight junction & 170 & 7.14 & 14 & 14 & 0.0121 & 0.175 \\
\hline Measles & 138 & 5.8 & 12 & 12 & 0.0131 & 0.181 \\
\hline Drug metabolism - other enzymes & 79 & 3.32 & 8 & 8 & 0.0174 & 0.23 \\
\hline Inflammatory bowel disease (IBD) & 65 & 2.73 & 7 & 7 & 0.0186 & 0.237 \\
\hline Folate biosynthesis & 26 & 1.09 & 4 & 4 & 0.022 & 0.269 \\
\hline Fanconianemia pathway & 54 & 2.27 & 6 & 6 & 0.0249 & 0.283 \\
\hline Ascorbate and aldarate metabolism & 27 & 1.13 & 4 & 4 & 0.025 & 0.283 \\
\hline Legionellosis & 55 & 2.31 & 6 & 6 & 0.0269 & 0.283 \\
\hline Bladder cancer & 41 & 1.72 & 5 & 5 & 0.0275 & 0.283 \\
\hline Apoptosis & 136 & 5.71 & 11 & 11 & 0.0276 & 0.283 \\
\hline Chemokine signaling pathway & 190 & 7.98 & 14 & 14 & 0.0288 & 0.286 \\
\hline Pyrimidine metabolism & 57 & 2.39 & 6 & 6 & 0.0315 & 0.303 \\
\hline Th17 cell differentiation & 107 & 4.49 & 9 & 9 & 0.0355 & 0.332 \\
\hline Pancreatic cancer & 75 & 3.15 & 7 & 7 & 0.0374 & 0.339 \\
\hline Steroid hormone biosynthesis & 60 & 2.52 & 6 & 6 & 0.0392 & 0.339 \\
\hline Alcoholism & 180 & 7.56 & 13 & 13 & 0.0395 & 0.339 \\
\hline Small cell lung cancer & 93 & 3.91 & 8 & 8 & 0.0411 & 0.342 \\
\hline Glycerolipid metabolism & 61 & 2.56 & 6 & 6 & 0.042 & 0.342 \\
\hline
\end{tabular}




\begin{tabular}{lllllll}
\hline Pathway & Total & Expected & Expected & Hits & P.Value & FDR \\
\hline Base excision repair & 33 & 1.39 & 4 & 4 & 0.0479 & 0.381 \\
One carbon pool by folate & 20 & 0.84 & 3 & 3 & 0.0493 & 0.382 \\
\hline
\end{tabular}

Covid patient BALF- GSA id PRJCA002326

\begin{tabular}{|c|c|c|c|c|c|c|}
\hline Pathway & Total & Expected & Expected & Hits & P.Value & FDR \\
\hline Alzheimer's disease & 171 & 71 & 104 & 104 & $2.10 \mathrm{E}-07$ & $5.03 \mathrm{E}-0$ \\
\hline Oxidative phosphorylation & 133 & 55.2 & 84 & 84 & $3.16 \mathrm{E}-07$ & $5.03 \mathrm{E}-0$ \\
\hline Parkinson's disease & 142 & 58.9 & 88 & 88 & $5.66 \mathrm{E}-07$ & $6.00 \mathrm{E}-0$ \\
\hline Chemokine signaling pathway & 190 & 78.9 & 105 & 105 & $7.50 \mathrm{E}-05$ & 0.00596 \\
\hline Malaria & 49 & 20.3 & 33 & 33 & 0.000221 & 0.0141 \\
\hline Dopaminergic synapse & 131 & 54.4 & 74 & 74 & 0.000346 & 0.0173 \\
\hline Phagosome & 152 & 63.1 & 84 & 84 & 0.000381 & 0.0173 \\
\hline Spliceosome & 134 & 55.6 & 75 & 75 & 0.000463 & 0.0184 \\
\hline Non-alcoholic fatty liver disease (NAFLD) & 149 & 61.8 & 82 & 82 & 0.00053 & 0.0187 \\
\hline Chagas disease (American trypanosomiasis) & 103 & 42.7 & 59 & 59 & 0.000832 & 0.0265 \\
\hline Notch signaling pathway & 48 & 19.9 & 31 & 31 & 0.00102 & 0.0295 \\
\hline Protein processing in endoplasmic reticulum & 165 & 68.5 & 88 & 88 & 0.00129 & 0.0316 \\
\hline Salmonella infection & 86 & 35.7 & 50 & 50 & 0.00129 & 0.0316 \\
\hline Platelet activation & 124 & 51.5 & 68 & 68 & 0.00173 & 0.0387 \\
\hline Pathogenic Escherichia coli infection & 55 & 22.8 & 34 & 34 & 0.00182 & 0.0387 \\
\hline Huntington's disease & 193 & 80.1 & 100 & 100 & 0.00218 & 0.0434 \\
\hline Pathways in cancer & 530 & 220 & 251 & 251 & 0.00274 & 0.051 \\
\hline Osteoclast differentiation & 128 & 53.1 & 69 & 69 & 0.00289 & 0.051 \\
\hline Fc gamma R-mediated phagocytosis & 91 & 37.8 & 51 & 51 & 0.00343 & 0.0575 \\
\hline Complement and coagulation cascades & 79 & 32.8 & 45 & 45 & 0.00383 & 0.0609 \\
\hline Oxytocin signaling pathway & 153 & 63.5 & 80 & 80 & 0.00423 & 0.064 \\
\hline Th1 and Th2 cell differentiation & 92 & 38.2 & 51 & 51 & 0.00464 & 0.0671 \\
\hline Epithelial cell signaling in Helicobacter pylori infection & 68 & 28.2 & 39 & 39 & 0.00586 & 0.0773 \\
\hline Hematopoietic cell lineage & 97 & 40.3 & 53 & 53 & 0.00587 & 0.0773 \\
\hline Leukocyte transendothelial migration & 112 & 46.5 & 60 & 60 & 0.00627 & 0.0773 \\
\hline Tight junction & 170 & 70.6 & 87 & 87 & 0.00632 & 0.0773 \\
\hline Apoptosis & 136 & 56.4 & 71 & 71 & 0.00711 & 0.0838 \\
\hline FoxOsignaling pathway & 132 & 54.8 & 69 & 69 & 0.0076 & 0.0863 \\
\hline Mitophagy - animal & 65 & 27 & 37 & 37 & 0.00845 & 0.0882 \\
\hline Shigellosis & 65 & 27 & 37 & 37 & 0.00845 & 0.0882 \\
\hline Prion diseases & 35 & 14.5 & 22 & 22 & 0.00867 & 0.0882 \\
\hline Primary immunodeficiency & 37 & 15.4 & 23 & 23 & 0.00888 & 0.0882 \\
\hline Epstein-Barr virus infection & 201 & 83.4 & 100 & 100 & 0.0102 & 0.0932 \\
\hline Pertussis & 76 & 31.5 & 42 & 42 & 0.0104 & 0.0932 \\
\hline Focal adhesion & 199 & 82.6 & 99 & 99 & 0.0105 & 0.0932 \\
\hline Calcium signaling pathway & 188 & 78 & 94 & 94 & 0.0106 & 0.0932 \\
\hline Amphetamine addiction & 68 & 28.2 & 38 & 38 & 0.0114 & 0.098 \\
\hline Vibrio cholerae infection & 50 & 20.8 & 29 & 29 & 0.0134 & 0.112 \\
\hline Regulation of actin cytoskeleton & 214 & 88.8 & 105 & 105 & 0.0141 & 0.113 \\
\hline Retrograde endocannabinoid signaling & 148 & 61.4 & 75 & 75 & 0.0143 & 0.113 \\
\hline HTLV-I infection & 219 & 90.9 & 107 & 107 & 0.0153 & 0.119 \\
\hline Kaposi's sarcoma-associated herpesvirus infection & 186 & 77.2 & 92 & 92 & 0.016 & 0.119 \\
\hline Carbon metabolism & 116 & 48.1 & 60 & 60 & 0.016 & 0.119 \\
\hline
\end{tabular}




\begin{tabular}{lllllll}
\hline Pathway & Total & Expected & Expected & Hits & P.Value & FDR \\
\hline Endocytosis & 244 & 101 & 118 & 118 & 0.0164 & 0.119 \\
Phosphatidylinositol signaling system & 99 & 41.1 & 52 & 52 & 0.0168 & 0.119 \\
Legionellosis & 55 & 22.8 & 31 & 31 & 0.0182 & 0.126 \\
Chronic myeloid leukemia & 76 & 31.5 & 41 & 41 & 0.0187 & 0.126 \\
Proteasome & 45 & 18.7 & 26 & 26 & 0.0199 & 0.132 \\
Hippo signaling pathway -multiple species & 29 & 12 & 18 & 18 & 0.0203 & 0.132 \\
Proteoglycans in cancer & 201 & 83.4 & 98 & 98 & 0.021 & 0.134 \\
Ribosome & 153 & 63.5 & 76 & 76 & 0.0239 & 0.149 \\
Cellular senescence & 160 & 66.4 & 79 & 79 & 0.0255 & 0.156 \\
B cell receptor signaling pathway & 71 & 29.5 & 38 & 38 & 0.0267 & 0.16 \\
2-Oxocarboxylic acid metabolism & 18 & 7.47 & 12 & 12 & 0.0276 & 0.161 \\
Circadian entrainment & 97 & 40.3 & 50 & 50 & 0.0283 & 0.161 \\
Long-term potentiation & 67 & 27.8 & 36 & 36 & 0.0285 & 0.161 \\
ECM-receptor interaction & 82 & 34 & 43 & 43 & 0.0289 & 0.161 \\
Basal cell carcinoma & 63 & 26.1 & 34 & 34 & 0.0303 & 0.166 \\
Autophagy - animal & 128 & 53.1 & 64 & 64 & 0.0309 & 0.166 \\
Apelinsignaling pathway & 137 & 56.9 & 68 & 68 & 0.0319 & 0.169 \\
Cell adhesion molecules (CAMs) & 146 & 60.6 & 72 & 72 & 0.0328 & 0.171 \\
Adherens junction & 72 & 29.9 & 38 & 38 & 0.0344 & 0.173 \\
Arrhythmogenic right ventricular cardiomyopathy (ARVC) & 72 & 29.9 & 38 & 38 & 0.0344 & 0.173 \\
Natural killer cell mediated cytotoxicity & 131 & 54.4 & 65 & 65 & 0.0356 & 0.177 \\
Non-small cell lung cancer & 66 & 27.4 & 35 & 35 & 0.0381 & 0.186 \\
Glucagon signaling pathway & 103 & 42.7 & 52 & 52 & 0.0398 & 0.188 \\
Oocyte meiosis & 125 & 51.9 & 62 & 62 & 0.0398 & 0.188 \\
Cytokine-cytokine receptor interaction & 294 & 122 & 137 & 137 & 0.0407 & 0.188 \\
Hippo signaling pathway & 154 & 63.9 & 75 & 75 & 0.0408 & 0.188 \\
Endometrial cancer & 58 & 24.1 & 31 & 31 & 0.0436 & 0.198 \\
Fructose and mannose metabolism & 33 & 13.7 & 19 & 19 & 0.0455 & 0.204 \\
cGMP-PKG signaling pathway & 166 & 68.9 & 80 & 80 & 0.0462 & 0.204 \\
IL-17 signaling pathway & 93 & 38.6 & 47 & 47 & 0.0479 & 0.209 \\
Rheumatoid arthritis & 91 & 37.8 & 46 & 46 & 0.0497 & 0.214 \\
\hline
\end{tabular}

Covid-19 Lung (Autopsy)- GSE150316

\begin{tabular}{llllll}
\hline Pathway & Total & Expectd & Hits & P.Value & FDR \\
\hline Cellular senescence & 160 & 11.8 & 33 & $4.00 \mathrm{E}-08$ & $1.27 \mathrm{E}-05$ \\
HTLV-I infection & 219 & 16.1 & 38 & $4.51 \mathrm{E}-07$ & $6.97 \mathrm{E}-05$ \\
FoxO signaling pathway & 132 & 9.71 & 27 & $8.29 \mathrm{E}-07$ & $6.97 \mathrm{E}-05$ \\
TNF signaling pathway & 110 & 8.09 & 24 & $1.00 \mathrm{E}-06$ & $6.97 \mathrm{E}-05$ \\
Chagas disease (American trypanosomiasis) & 103 & 7.57 & 23 & $1.10 \mathrm{E}-06$ & $6.97 \mathrm{E}-05$ \\
Kaposi's sarcoma-associated herpesvirus infection & 186 & 13.7 & 33 & $1.57 \mathrm{E}-06$ & $8.32 \mathrm{E}-05$ \\
Pathways in cancer & 530 & 39 & 67 & $5.56 \mathrm{E}-06$ & 0.000253 \\
Renal cell carcinoma & 69 & 5.07 & 17 & $6.86 \mathrm{E}-06$ & 0.000273 \\
Colorectal cancer & 86 & 6.32 & 18 & $4.10 \mathrm{E}-05$ & 0.00138 \\
Complement and coagulation cascades & 79 & 5.81 & 17 & $4.60 \mathrm{E}-05$ & 0.00138 \\
p53 signaling pathway & 72 & 5.3 & 16 & $5.06 \mathrm{E}-05$ & 0.00138 \\
Apelin signaling pathway & 137 & 10.1 & 24 & $5.19 \mathrm{E}-05$ & 0.00138 \\
Insulin resistance & 108 & 7.94 & 20 & $9.79 \mathrm{E}-05$ & 0.00239 \\
MAPK signaling pathway & 295 & 21.7 & 40 & 0.000105 & 0.00239
\end{tabular}




\begin{tabular}{|c|c|c|c|c|c|}
\hline Pathway & Total & Expectd & Hits & P.Value & FDR \\
\hline mTOR signaling pathway & 153 & 11.3 & 25 & 0.000118 & 0.00239 \\
\hline IL-17 signaling pathway & 93 & 6.84 & 18 & 0.00012 & 0.00239 \\
\hline Hepatitis B & 163 & 12 & 26 & 0.000131 & 0.00245 \\
\hline cAMP signaling pathway & 212 & 15.6 & 31 & 0.000161 & 0.00285 \\
\hline cGMP-PKG signaling pathway & 166 & 12.2 & 26 & 0.000177 & 0.00297 \\
\hline Circadian rhythm & 31 & 2.28 & 9 & 0.000274 & 0.00436 \\
\hline Oxytocin signaling pathway & 153 & 11.3 & 24 & 0.000305 & 0.00451 \\
\hline AGE-RAGE signaling pathway in diabetic complications & 100 & 7.35 & 18 & 0.000312 & 0.00451 \\
\hline Proteoglycans in cancer & 201 & 14.8 & 29 & 0.000329 & 0.00455 \\
\hline Chronic myeloid leukemia & 76 & 5.59 & 15 & 0.00035 & 0.00464 \\
\hline Sphingolipid signaling pathway & 119 & 8.75 & 20 & 0.000382 & 0.00485 \\
\hline Breast cancer & 147 & 10.8 & 23 & 0.00042 & 0.00514 \\
\hline B cell receptor signaling pathway & 71 & 5.22 & 14 & 0.000553 & 0.00651 \\
\hline Prostate cancer & 97 & 7.13 & 17 & 0.000627 & 0.00691 \\
\hline Melanoma & 72 & 5.3 & 14 & 0.000641 & 0.00691 \\
\hline Platelet activation & 124 & 9.12 & 20 & 0.000661 & 0.00691 \\
\hline Longevity regulating pathway & 89 & 6.55 & 16 & 0.000673 & 0.00691 \\
\hline Mitophagy - animal & 65 & 4.78 & 13 & 0.00075 & 0.00746 \\
\hline Ras signaling pathway & 232 & 17.1 & 31 & $8.00 \mathrm{E}-04$ & 0.00771 \\
\hline Rheumatoid arthritis & 91 & 6.69 & 16 & 0.000866 & 0.008 \\
\hline Apoptosis & 136 & 10 & 21 & 0.000881 & 0.008 \\
\hline Pancreatic cancer & 75 & 5.52 & 14 & 0.00098 & 0.00842 \\
\hline Glioma & 75 & 5.52 & 14 & 0.00098 & 0.00842 \\
\hline Neurotrophin signaling pathway & 119 & 8.75 & 19 & 0.00101 & 0.00848 \\
\hline Small cell lung cancer & 93 & 6.84 & 16 & 0.0011 & 0.00892 \\
\hline Pertussis & 76 & 5.59 & 14 & 0.00112 & 0.00892 \\
\hline Epithelial cell signaling in Helicobacter pylori infection & 68 & 5 & 13 & 0.00117 & 0.00907 \\
\hline Legionellosis & 55 & 4.04 & 11 & 0.00188 & 0.014 \\
\hline Th17 cell differentiation & 107 & 7.87 & 17 & 0.00194 & 0.014 \\
\hline Regulation of actin cytoskeleton & 214 & 15.7 & 28 & 0.00194 & 0.014 \\
\hline Phosphatidylinositol signaling system & 99 & 7.28 & 16 & 0.00217 & 0.015 \\
\hline Choline metabolism in cancer & 99 & 7.28 & 16 & 0.00217 & 0.015 \\
\hline Rap1 signaling pathway & 206 & 15.1 & 27 & 0.00226 & 0.0153 \\
\hline Insulin signaling pathway & 137 & 10.1 & 20 & 0.00232 & 0.0154 \\
\hline Non-small cell lung cancer & 66 & 4.85 & 12 & 0.00279 & 0.018 \\
\hline Focal adhesion & 199 & 14.6 & 26 & 0.00283 & 0.018 \\
\hline Endometrial cancer & 58 & 4.27 & 11 & 0.00293 & 0.0183 \\
\hline Chemokine signaling pathway & 190 & 14 & 25 & 0.00308 & 0.0188 \\
\hline Prion diseases & 35 & 2.57 & 8 & 0.00324 & 0.0195 \\
\hline ErbB signaling pathway & 85 & 6.25 & 14 & 0.00336 & 0.0198 \\
\hline Salmonella infection & 86 & 6.32 & 14 & 0.00376 & 0.0217 \\
\hline Adipocytokine signaling pathway & 69 & 5.07 & 12 & 0.00408 & 0.023 \\
\hline Amoebiasis & 96 & 7.06 & 15 & 0.00412 & 0.023 \\
\hline EGFR tyrosine kinase inhibitor resistance & 79 & 5.81 & 13 & 0.00468 & 0.0256 \\
\hline Cytokine-cytokine receptor interaction & 294 & 21.6 & 34 & 0.00532 & 0.0287 \\
\hline Wnt signaling pathway & 158 & 11.6 & 21 & 0.00569 & 0.0297 \\
\hline Measles & 138 & 10.1 & 19 & 0.00569 & 0.0297 \\
\hline HIF-1 signaling pathway & 100 & 7.35 & 15 & 0.00607 & 0.0306 \\
\hline Inflammatory mediator regulation of TRP channels & 100 & 7.35 & 15 & 0.00607 & 0.0306 \\
\hline Fluid shear stress and atherosclerosis & 139 & 10.2 & 19 & 0.00615 & 0.0306 \\
\hline
\end{tabular}




\begin{tabular}{|c|c|c|c|c|c|}
\hline Pathway & Total & Expectd & Hits & P.Value & FDR \\
\hline PI3K-Akt signaling pathway & 354 & 26 & 39 & 0.00673 & 0.0329 \\
\hline Cholinergic synapse & 112 & 8.24 & 16 & 0.00754 & 0.0362 \\
\hline GnRH signaling pathway & 93 & 6.84 & 14 & 0.00764 & 0.0362 \\
\hline Vascular smooth muscle contraction & 132 & 9.71 & 18 & 0.00774 & 0.0362 \\
\hline Glucagon signaling pathway & 103 & 7.57 & 15 & 0.00799 & 0.0368 \\
\hline Malaria & 49 & 3.6 & 9 & 0.00853 & 0.0387 \\
\hline Fc epsilon RI signaling pathway & 68 & 5 & 11 & 0.0101 & 0.0448 \\
\hline Amphetamine addiction & 68 & 5 & 11 & 0.0101 & 0.0448 \\
\hline Pantothenate and CoA biosynthesis & 19 & 1.4 & 5 & 0.0103 & 0.045 \\
\hline Aldosterone synthesis and secretion & 98 & 7.21 & 14 & 0.012 & 0.0505 \\
\hline Estrogen signaling pathway & 138 & 10.1 & 18 & 0.0121 & 0.0505 \\
\hline Autophagy - animal & 128 & 9.41 & 17 & 0.0122 & 0.0505 \\
\hline Osteoclast differentiation & 128 & 9.41 & 17 & 0.0122 & 0.0505 \\
\hline Prolactin signaling pathway & 70 & 5.15 & 11 & 0.0125 & 0.0511 \\
\hline Signaling pathways regulating pluripotency of stem cells & 139 & 10.2 & 18 & 0.013 & 0.0522 \\
\hline Axon guidance & 181 & 13.3 & 22 & 0.0132 & 0.0524 \\
\hline Longevity regulating pathway - multiple species & 62 & 4.56 & 10 & 0.0142 & 0.0554 \\
\hline AMPK signaling pathway & 120 & 8.83 & 16 & 0.0143 & 0.0554 \\
\hline $\mathrm{T}$ cell receptor signaling pathway & 101 & 7.43 & 14 & 0.0154 & 0.0584 \\
\hline Melanogenesis & 101 & 7.43 & 14 & 0.0154 & 0.0584 \\
\hline Thyroid cancer & 37 & 2.72 & 7 & 0.0166 & 0.0623 \\
\hline Transcriptional misregulation in cancer & 186 & 13.7 & 22 & 0.0177 & 0.0654 \\
\hline Leishmaniasis & 74 & 5.44 & 11 & 0.0186 & 0.068 \\
\hline Adrenergic signaling in cardiomyocytes & 145 & 10.7 & 18 & 0.0194 & 0.0694 \\
\hline Central carbon metabolism in cancer & 65 & 4.78 & 10 & 0.0194 & 0.0694 \\
\hline Epstein-Barr virus infection & 201 & 14.8 & 23 & 0.0222 & 0.0783 \\
\hline Phospholipase D signaling pathway & 148 & 10.9 & 18 & 0.0234 & 0.0817 \\
\hline Hematopoietic cell lineage & 97 & 7.13 & 13 & 0.0247 & 0.0855 \\
\hline Gap junction & 88 & 6.47 & 12 & 0.0268 & 0.0917 \\
\hline Bladder cancer & 41 & 3.02 & 7 & 0.0282 & 0.0955 \\
\hline Progesterone-mediated oocyte maturation & 99 & 7.28 & 13 & 0.0287 & 0.0962 \\
\hline Dopaminergic synapse & 131 & 9.63 & 16 & 0.0303 & 0.1 \\
\hline NF-kappa B signaling pathway & 100 & 7.35 & 13 & 0.0309 & 0.101 \\
\hline Hippo signaling pathway & 154 & 11.3 & 18 & 0.0332 & 0.108 \\
\hline Leukocyte transendothelial migration & 112 & 8.24 & 14 & 0.0346 & 0.111 \\
\hline Fatty acid biosynthesis & 18 & 1.32 & 4 & 0.0387 & 0.123 \\
\hline Platinum drug resistance & 73 & 5.37 & 10 & 0.04 & 0.126 \\
\hline Toll-like receptor signaling pathway & 104 & 7.65 & 13 & 0.0408 & 0.127 \\
\hline Thyroid hormone signaling pathway & 116 & 8.53 & 14 & 0.0447 & 0.138 \\
\hline Non-alcoholic fatty liver disease (NAFLD) & 149 & 11 & 17 & 0.046 & 0.14 \\
\hline Hypertrophic cardiomyopathy (HCM) & 85 & 6.25 & 11 & 0.0461 & 0.14 \\
\hline Shigellosis & 65 & 4.78 & 9 & 0.0471 & 0.141 \\
\hline
\end{tabular}

\section{DIOD rodent lung 1- GSE123938}

\begin{tabular}{llllll}
\hline Pathway & Total & Expected & Hits & P.Value & FDR \\
\hline Primary immunodeficiency & 36 & 1.77 & 12 & $7.12 \mathrm{E}-08$ & $2.24 \mathrm{E}-05$ \\
Hematopoietic cell lineage & 95 & 4.66 & 15 & $5.34 \mathrm{E}-05$ & 0.00838 \\
Tryptophan metabolism & 48 & 2.36 & 9 & 0.00046 & 0.0482
\end{tabular}




\begin{tabular}{llllll}
\hline Pathway & Total & Expected & Hits & P.Value & FDR \\
\hline Cytokine-cytokine receptor interaction & 296 & 14.5 & 28 & 0.000622 & 0.0488 \\
Morphine addiction & 92 & 4.51 & 12 & 0.00171 & 0.107 \\
Jak-STAT signaling pathway & 165 & 8.1 & 17 & 0.00296 & 0.139 \\
AMPK signaling pathway & 126 & 6.18 & 14 & 0.00341 & 0.139 \\
cAMPsignaling pathway & 211 & 10.4 & 20 & 0.00354 & 0.139 \\
Intestinal immune network for IgA production & 43 & 2.11 & 7 & 0.00453 & 0.15 \\
Wntsignaling pathway & 160 & 7.85 & 16 & 0.0052 & 0.15 \\
PI3K-Akt signaling pathway & 358 & 17.6 & 29 & 0.00526 & 0.15 \\
Rheumatoid arthritis & 84 & 4.12 & 10 & 0.00777 & 0.203 \\
Cholinergic synapse & 113 & 5.54 & 12 & 0.00923 & 0.223 \\
Th17 cell differentiation & 102 & 5.01 & 11 & 0.0111 & 0.229 \\
Aldosterone synthesis and secretion & 102 & 5.01 & 11 & 0.0111 & 0.229 \\
Neuroactive ligand-receptor interaction & 348 & 17.1 & 27 & 0.012 & 0.229 \\
GABAergic synapse & 90 & 4.42 & 10 & 0.0124 & 0.229 \\
Purine metabolism & 136 & 6.67 & 13 & 0.0159 & 0.271 \\
Amphetamine addiction & 68 & 3.34 & 8 & 0.0176 & 0.271 \\
HTLV-I infection & 246 & 12.1 & 20 & 0.0181 & 0.271 \\
Apelinsignaling pathway & 139 & 6.82 & 13 & 0.0188 & 0.271 \\
Hedgehog signaling pathway & 44 & 2.16 & 6 & 0.0196 & 0.271 \\
Cell adhesion molecules (CAMs) & 171 & 8.39 & 15 & 0.0207 & 0.271 \\
Fatty acid metabolism & 57 & 2.8 & 7 & 0.0207 & 0.271 \\
Circadian entrainment & 99 & 4.86 & 10 & 0.023 & 0.281 \\
Calcium signaling pathway & 189 & 9.27 & 16 & 0.0233 & 0.281 \\
T cell receptor signaling pathway & 101 & 4.96 & 10 & 0.026 & 0.295 \\
Th1 and Th2 cell differentiation & 87 & 4.27 & 9 & 0.0263 & 0.295 \\
ABC transporters & 48 & 2.36 & 6 & 0.0289 & 0.313 \\
Dilated cardiomyopathy & 90 & 4.42 & 9 & 0.0319 & 0.334 \\
Dopaminergic synapse & 135 & 6.62 & 12 & 0.0331 & 0.335 \\
Focal adhesion & 199 & 9.77 & 16 & 0.0353 & 0.346 \\
Butanoate metabolism & 27 & 1.32 & 4 & 0.041 & 0.379 \\
Phototransduction & 27 & 1.32 & 4 & 0.041 & 0.379 \\
Selenocompound metabolism & 17 & 0.834 & 3 & 0.0478 & 0.429 \\
\hline & & & & &
\end{tabular}

DIOD rodent lung 2- GSE3963412

\begin{tabular}{llllll}
\hline Pathway & Total & Expected & Hits & P.Value & FDR \\
\hline Hematopoietic cell lineage & 95 & 7.1 & 27 & $6.64 \mathrm{E}-10$ & $1.46 \mathrm{E}-07$ \\
B cell receptor signaling pathway & 72 & 5.38 & 23 & $9.83 \mathrm{E}-10$ & $1.46 \mathrm{E}-07$ \\
Primary immunodeficiency & 36 & 2.69 & 16 & $1.40 \mathrm{E}-09$ & $1.46 \mathrm{E}-07$ \\
Protein processing in endoplasmic reticulum & 163 & 12.2 & 30 & $3.07 \mathrm{E}-06$ & 0.000241 \\
T cell receptor signaling pathway & 101 & 7.55 & 22 & $3.85 \mathrm{E}-06$ & 0.000242 \\
Calcium signaling pathway & 189 & 14.1 & 30 & $6.11 \mathrm{E}-05$ & 0.0032 \\
NF-kappa B signaling pathway & 109 & 8.14 & 20 & 0.000141 & 0.00607 \\
Cell adhesion molecules (CAMs) & 171 & 12.8 & 27 & 0.000155 & 0.00607 \\
Epstein-Barr virus infection & 230 & 17.2 & 33 & $2.00 \mathrm{E}-04$ & 0.00696 \\
Antigen processing and presentation & 91 & 6.8 & 17 & 0.000351 & 0.011 \\
Chemokine signaling pathway & 200 & 14.9 & 29 & 0.000401 & 0.0114 \\
AGE-RAGE signaling pathway in diabetic complications & 101 & 7.55 & 18 & 0.000434 & 0.0114 \\
Fc gamma R-mediated phagocytosis & 87 & 6.5 & 16 & 0.000622 & 0.015
\end{tabular}




\begin{tabular}{|c|c|c|c|c|c|}
\hline Pathway & Total & Expected & Hits & P.Value & FDR \\
\hline Leukocyte transendothelial migration & 115 & 8.59 & 19 & 0.000808 & 0.0181 \\
\hline Leishmaniasis & 67 & 5.01 & 13 & 0.00118 & 0.0232 \\
\hline Jak-STAT signaling pathway & 165 & 12.3 & 24 & 0.00118 & 0.0232 \\
\hline HIF-1 signaling pathway & 105 & 7.85 & 17 & 0.00188 & 0.0347 \\
\hline EGFR tyrosine kinase inhibitor resistance & 80 & 5.98 & 14 & 0.00219 & 0.0382 \\
\hline Toxoplasmosis & 108 & 8.07 & 17 & 0.00256 & 0.0418 \\
\hline Natural killer cell mediated cytotoxicity & 118 & 8.82 & 18 & 0.00277 & 0.0418 \\
\hline Non-small cell lung cancer & 66 & 4.93 & 12 & 0.00319 & 0.0418 \\
\hline Legionellosis & 58 & 4.33 & 11 & 0.00333 & 0.0418 \\
\hline Arginine and proline metabolism & 50 & 3.74 & 10 & 0.00337 & 0.0418 \\
\hline ErbBsignaling pathway & 84 & 6.28 & 14 & 0.00349 & 0.0418 \\
\hline Th17 cell differentiation & 102 & 7.62 & 16 & 0.0035 & 0.0418 \\
\hline Focal adhesion & 199 & 14.9 & 26 & 0.00355 & 0.0418 \\
\hline Pathways in cancer & 535 & 40 & 57 & 0.00363 & 0.0418 \\
\hline Mucin type O-glycan biosynthesis & 28 & 2.09 & 7 & 0.00372 & 0.0418 \\
\hline Intestinal immune network for IgA production & 43 & 3.21 & 9 & 0.00387 & 0.0419 \\
\hline Cytokine-cytokine receptor interaction & 296 & 22.1 & 35 & 0.00433 & 0.0453 \\
\hline Measles & 144 & 10.8 & 20 & 0.00503 & 0.051 \\
\hline NOD-like receptor signaling pathway & 206 & 15.4 & 26 & 0.00565 & 0.0539 \\
\hline Longevity regulating pathway - multiple species & 62 & 4.63 & 11 & 0.00567 & 0.0539 \\
\hline GABAergic synapse & 90 & 6.73 & 14 & 0.00658 & 0.0608 \\
\hline Pancreatic cancer & 75 & 5.6 & 12 & 0.00917 & 0.0823 \\
\hline Glutamatergic synapse & 114 & 8.52 & 16 & 0.0104 & 0.0899 \\
\hline Regulation of actin cytoskeleton & 217 & 16.2 & 26 & 0.011 & 0.0899 \\
\hline Platinum drug resistance & 77 & 5.75 & 12 & 0.0113 & 0.0899 \\
\hline Platelet activation & 125 & 9.34 & 17 & 0.0114 & 0.0899 \\
\hline Prion diseases & 34 & 2.54 & 7 & 0.0115 & 0.0899 \\
\hline Th1 and Th2 cell differentiation & 87 & 6.5 & 13 & 0.012 & 0.0922 \\
\hline Rap1 signaling pathway & 209 & 15.6 & 25 & 0.0127 & 0.0931 \\
\hline Glycosphingolipid biosynthesis - lacto and neolacto series & 27 & 2.02 & 6 & 0.013 & 0.0931 \\
\hline Carbohydrate digestion and absorption & 43 & 3.21 & 8 & 0.013 & 0.0931 \\
\hline Osteoclast differentiation & 128 & 9.56 & 17 & 0.0142 & 0.0992 \\
\hline Axon guidance & 180 & 13.5 & 22 & 0.0148 & 0.101 \\
\hline Protein digestion and absorption & 90 & 6.73 & 13 & 0.0158 & 0.105 \\
\hline PI3K-Akt signaling pathway & 358 & 26.8 & 38 & 0.017 & 0.111 \\
\hline MAPK signaling pathway & 294 & 22 & 32 & 0.0196 & 0.126 \\
\hline Alanine, aspartate and glutamate metabolism & 38 & 2.84 & 7 & 0.0208 & 0.13 \\
\hline Estrogensignaling pathway & 134 & 10 & 17 & 0.0216 & 0.133 \\
\hline Sphingolipid metabolism & 48 & 3.59 & 8 & 0.0245 & 0.148 \\
\hline Rassignaling pathway & 233 & 17.4 & 26 & 0.0252 & 0.148 \\
\hline Amoebiasis & 106 & 7.92 & 14 & 0.0257 & 0.148 \\
\hline Nicotine addiction & 40 & 2.99 & 7 & 0.027 & 0.148 \\
\hline VEGF signaling pathway & 58 & 4.33 & 9 & 0.027 & 0.148 \\
\hline Endometrial cancer & 58 & 4.33 & 9 & 0.027 & 0.148 \\
\hline Glycosphingolipid biosynthesis - globo and isoglobo series & 16 & 1.2 & 4 & 0.0273 & 0.148 \\
\hline Prostate cancer & 97 & 7.25 & 13 & 0.0278 & 0.148 \\
\hline Renal cell carcinoma & 68 & 5.08 & 10 & 0.0286 & 0.15 \\
\hline Inflammatory bowel disease (IBD) & 59 & 4.41 & 9 & 0.0298 & 0.154 \\
\hline Fluid shear stress and atherosclerosis & 143 & 10.7 & 17 & 0.0375 & 0.187 \\
\hline IL-17 signaling pathway & 91 & 6.8 & 12 & 0.0376 & 0.187 \\
\hline
\end{tabular}




\begin{tabular}{llllll}
\hline Pathway & Total & Expected & Hits & P.Value & FDR \\
\hline Small cell lung cancer & 92 & 6.87 & 12 & 0.0404 & 0.196 \\
Adherens junction & 72 & 5.38 & 10 & 0.0405 & 0.196 \\
Cholinergic synapse & 113 & 8.44 & 14 & 0.0415 & 0.197 \\
Chagas disease (American trypanosomiasis) & 103 & 7.7 & 13 & 0.0426 & 0.198 \\
Sphingolipid signaling pathway & 124 & 9.27 & 15 & 0.0428 & 0.198 \\
Thyroid hormone signaling pathway & 115 & 8.59 & 14 & 0.047 & 0.213 \\
Gastric acid secretion & 74 & 5.53 & 10 & 0.0475 & 0.213 \\
Phagosome & 181 & 13.5 & 20 & 0.0497 & 0.22 \\
\hline
\end{tabular}

